\title{
DISTANCE FOR BÉZIER CURVES AND DEGREE REDUCTION
}

\author{
Byung-Gook Lee ANd Yunbeom Park
}

An algorithmic approach to degree reduction of Bézier curves is presented. The algorithm is based on the matrix representations of the degree elevation and degree reduction processes. The control points of the approximation are obtained by the generalised least squares method. The computations are carried out by minimising the $L_{2}$ and discrete $l_{2}$ distance between the two curves.

\section{INTRODUCTION}

Bézier curves are basically and widely used in CAGD - short for Computer Aided Geometric Design. Bézier curves were independently developed by de Casteljau about 1959 [2] and by Bézier about 1962 [1]. The underlying mathematical theory is based on the concept of Bernstein polynomials. De Casteljau directly exploited this relationship; but it was not until 1970 that Forrest[11] discovered the connection between Bézier's work and Bernstein polynomials. Bézier and de Casteljau developed their theories as part of CAD systems that were being built up at two French car companies, Rénault and Citroën. The Rénault system UNISURF (by Bézier) was soon described in several publications; this is the reason that the underlying theory now bears Bézier's name. Bézier curves and surfaces are now established as the mathematical basis of many CAD systems, they have also become a major tool for the development of new methods for curve and surface descriptions. Farin [10] summarises the basic theory of such curves and provides many relevant references.

The Bézier representation uses Bernstein polynomials as basis functions for the linear space of polynomials. In terms of the Bernstein polynomials of degree $n$,

$$
B_{k}^{n}(t)=\left(\begin{array}{l}
n \\
k
\end{array}\right)(1-t)^{n-k} t^{k}, 0 \leqslant t \leqslant 1, k=0, \cdots, n,
$$

a parametric polynomial curve of degree $n(n>0)$ in the plane, can be expressed as

$$
b^{n}(t)=\sum_{k=0}^{n} b_{k} B_{k}^{n}(t), b_{k} \in \mathbb{R}^{2}
$$

Received 25th March, 1997

This paper was supported by non directed research fund, Korea Research Foundation, 1996.

Copyright Clearance Centre, Inc. Serial-fee code: 0004-9729/97 \$A2.00+0.00. 
The points $b_{k}, k=0, \cdots, n$ are called the control points for the polynomial, and the polygon formed by joining successive control points is the control polygon. Notice that $b_{0}$ and $b_{n}$ are the endpoints of the curve corresponding to $t=0$ and $t=1$; we shall refer to these particular points as anchor points. Moreover, the vector $b_{1}-b_{0}$ and $b_{n}-b_{n-1}$ define the tangents to the curve at the two anchor points respectively.

In general, degree reduction of Bézier curves address the following problem.

Problem 1. (Degree Reduction.) Let $\left\{b_{i}\right\}_{i=0}^{n} \subset \mathbb{R}^{2}$ be a given set of control points which define the Bézier curve

$$
b^{n}(t)=\sum_{i=0}^{n} b_{i} B_{i}^{n}(t), \quad 0 \leqslant t \leqslant 1
$$

of degree $n$. Then find another point set $\left\{c_{i}\right\}_{i=0}^{m} \subset \mathbb{R}^{2}$ defining the approximative Bézier curve

$$
c^{m}(t)=\sum_{i=0}^{m} c_{i} B_{i}^{m}(t), \quad 0 \leqslant t \leqslant 1
$$

of lower degree $m<n$ so that a suitable distance function $d\left(b^{n}, c^{m}\right)$ between $b^{n}$ and $c^{m}$ is minimised.

In the literature $[3,4,6,7,9,11,14,15,17,19]$ one can find several schemes producing solutions for this approximation problem. These schemes mainly differ in the choice of the distance function and requiring the solution to be either best or only nearly best relative to the distance function. For instance, one special type of degree reduction schemes works recursively by lowering the degree only by one in every step - a procedure commonly known as economisation.

Examples for such a stepwise method were recently given in [6] or [19] where a very simple geometric construction of the new control points in each step is described. And the method allows detailed error analysis for the other methods (for example, [11] and $[9]$, see [18]). However, this general construction contains some scalar - valued degrees of freedom which are then chosen in such a way that the maximal Euclidean distance

$$
d_{\infty}\left(b^{n}, c^{n-1}\right)=\max _{0 \leqslant t \leqslant 1}\left\|b^{n}(t)-c^{n-1}(t)\right\|
$$

between two curves with respect to the given parameterisation is minimisied.

The derivation is mainly based on the so-called constrained Chebyshev polynomials. Unfortunately, the constrained Chebyshev polynomials are not known explicitly so their coefficients have to be determined numerically, which itself needs a lot of implementation effort.

This major disadvantage is avoided in the current paper. In more detail, we minimisie the least squares distance function

$$
d_{2}\left(b^{n}, c^{m}\right)=\sqrt{\int_{0}^{1}\left\|b^{n}(t)-c^{m}(t)\right\|^{2} d t}
$$


The algorithm presented is faster, more stable and much easier to implement. Moreover, the procedure can reduce the degree from $n$ to $m$ in only one step.

\section{Degree ELEVATION AND $L_{2}$ DISTANCE}

Suppose we were designing with Bézier curves trying to use a Bézier curve of degree $n$. After modifying the polygon a few times, it may turn out that a degree $n$ curve does not possess sufficient flexibility to model the desired shape. One way to proceed in such a situation is to increase the flexibility of the polygon by adding another vertex to it. As a first step, one might want to add another vertex yet leave the shape of the curve unchanged - this corresponds to raising the degree of the Bézier curve by one. We can show that new vertices $b_{i}^{(1)}$ are obtained from the old polygon by piecewise linear interpolation at the parameter values $i /(n+1)$

$$
b_{i}^{(1)}=\frac{i}{n+1} b_{i-1}+\left(1-\frac{i}{n+1}\right) b_{i}, i=0,1, \ldots, n+1 \text {. }
$$

We can rewrite the formula (1) as a linear system $T_{n} B=B^{(1)}$, where the $(n+2) \times$ $(n+1)$ matrix $T_{n}$ is

$$
T_{n}=\frac{1}{n+1}\left(\begin{array}{ccccccc}
n+1 & 0 & 0 & \ldots & 0 & 0 & 0 \\
1 & n & 0 & \ldots & 0 & 0 & 0 \\
0 & 2 & n-1 & \ldots & 0 & 0 & 0 \\
\vdots & \vdots & \vdots & \ddots & \vdots & \vdots & \vdots \\
0 & 0 & 0 & \ldots & n-1 & 2 & 0 \\
0 & 0 & 0 & \ldots & 0 & n & 1 \\
0 & 0 & 0 & \ldots & 0 & 0 & n+1
\end{array}\right)
$$

and the $(n+1)$ vector $B$ and the $(n+2)$ vector $B^{(1)}$ are

$$
\begin{aligned}
B & =\left(b_{0}, b_{1}, \ldots, b_{n}\right)^{t} \\
B^{(1)} & =\left(b_{0}^{(1)}, b_{1}^{(1)}, \ldots, b_{n+1}^{(1)}\right)^{t} .
\end{aligned}
$$

We may repeat this process and then obtain a sequence of control points. After $r$ degree elevations, we have a linear system $T_{n, r} B=B^{(r)}$, where the $(n+r+1) \times(n+1)$ matrix

$$
T_{n, r}=T_{n+r-1} T_{n+r-2} \ldots T_{n+1} T_{n}
$$

has elements

$$
t_{i+j, i}=\frac{\left(\begin{array}{c}
n \\
i
\end{array}\right)\left(\begin{array}{c}
r \\
j
\end{array}\right)}{\left(\begin{array}{c}
n+r \\
i+j
\end{array}\right)}, \quad\left\{\begin{array}{l}
i=0,1, \ldots, n \\
j=0,1, \ldots, r
\end{array}\right.
$$


The sum of any row and any column of the matrix $T_{n, r}$ are 1 and $(n+r+1) /(n+1)$ respectively, that is, for any $i$,

$$
\sum_{k=0}^{n} t_{i, k}=1
$$

and for any $k$,

$$
\sum_{i=0}^{n+r} t_{i, k}=\frac{n+r+1}{n+1} .
$$

For the degree reduction of any given curves, we must compute the distance of two Bézier curves. The most appropriate metric in geometrical terms would be the Hausdorff distance [5]. Suppose $(M, d)$ is a metric space with subsets $A$ and $B$. We define the Hausdorff metric $d_{H}$ by

$$
d_{H}(A, B)=\max \left\{\sup _{x \in A} d(x, B), \sup _{y \in B} d(y, A)\right\},
$$

where

$$
d(x, B)=\inf _{y \in B} d(x, y)
$$

If we regard a plane curve as simply a locus of points without any underlying parameterisation, the Hausdorff metric for two such curves is essentially the radius of the largest circle with its centre on one curve and touching the other curve. For general parametric curves, this measure is truly independent of the relative parameterisations of two curves. Emery [8] presents a method for explicit computation of the Hausdorff metric for piecewise linear curves, but the computation of the Hausdorff distance $d_{H}$ of two nonlinear curves is not so easy. So we define and use the $L_{2}$ distance for the Bézier curves.

We first consider the functional case of Bézier curves for computation of the $L_{2}$ distance of the two Bézier curves. Let $a^{n}$ and $b^{m}$ be functional Bézier curves of degree $n$ and $m(m<n)$, that is,

$$
a^{n}(t)=\sum_{k=0}^{n} a_{k} B_{k}^{n}(t), \quad b^{m}(t)=\sum_{k=0}^{m} b_{k} B_{k}^{m}(t),
$$

where the coefficients $a_{k}$ and $b_{k}$ are real numbers. The $L_{2}$ distance of the two Bézier curves $a^{n}$ and $b^{m}$ is defined as following:

$$
\begin{aligned}
d_{2}\left(a^{n}, b^{m}\right) & =\left\{\int_{0}^{1}\left|a^{n}(t)-b^{m}(t)\right|^{2} d t\right\}^{1 / 2} \\
& =\left\{\int_{0}^{1}\left|\sum_{k=0}^{n} a_{k} B_{k}^{n}(t)-\sum_{k=0}^{m} b_{k} B_{k}^{m}(t)\right|^{2} d t\right\}^{1 / 2}
\end{aligned}
$$

Using the matrix $T_{m, r}$, we can elevate the degree of $b^{m}$ from $m$ to $n$,

$$
B^{(r)}=T_{m, r} B
$$


Then, the curve $b^{m}$ of degree $m$ is re-written as a curve of degree $n$

$$
b^{m}(t)=b^{(r)}(t)=\sum_{k=0}^{n} b_{k}^{(r)} B_{k}^{n}(t),
$$

and the distance is

$$
\begin{aligned}
d_{2}\left(a^{n}, b^{m}\right) & =d_{2}\left(a^{n}, b^{(r)}\right) \\
& =\left\{\int_{0}^{1}\left|\sum_{k=0}^{n} a_{k} B_{k}^{n}(t)-\sum_{k=0}^{n} b_{k}^{(r)} B_{k}^{n}(t)\right|^{2} d t\right\}^{1 / 2} \\
& =\left\{\int_{0}^{1}\left|\sum_{k=0}^{n}\left(a_{k}-b_{k}^{(r)}\right) B_{k}^{n}(t)\right|^{2} d t\right\}^{1 / 2} .
\end{aligned}
$$

Let $c_{k}=a_{k}-b_{k}^{(r)}$ for all $k$. This is how we compute the $L_{2}$ norm of a (functional) Bézier curve of degree $n$.

The product of Bernstein polynomials is

$$
B_{i}^{n}(t) B_{j}^{m}(t)=\frac{\left(\begin{array}{c}
n \\
i
\end{array}\right)\left(\begin{array}{c}
m \\
j
\end{array}\right)}{\left(\begin{array}{c}
n+m \\
i+j
\end{array}\right)} B_{i+j}^{n+m}(t)
$$

and the integration is

$$
\int_{0}^{1} B_{k}^{n}(t) d t=\frac{1}{n+1}
$$

From these equations (2) and (3), we obtain the following computation for the $L_{2}$ norm of the functional Bézier curve $c^{n}$ :

$$
\begin{aligned}
\left\|c^{n}\right\|_{2}^{2} & =\int_{0}^{1}\left|\sum_{k=0}^{n} c_{k} B_{k}^{n}(t)\right|^{2} d t \\
& =\int_{0}^{1} \sum_{i, j} c_{i} c_{j} B_{i}^{n}(t) B_{j}^{n}(t) d t \\
& =\sum_{i, j} c_{i} c_{j} \int_{0}^{1} \frac{\left(\begin{array}{c}
n \\
i
\end{array}\right)\left(\begin{array}{l}
n \\
j
\end{array}\right)}{\left(\begin{array}{c}
2 n \\
i+j
\end{array}\right)} B_{i+j}^{2 n}(t) d t \\
& =\frac{1}{2 n+1} \sum_{i, j} c_{i} c_{j} \frac{\left(\begin{array}{c}
n \\
i
\end{array}\right)\left(\begin{array}{c}
n \\
j
\end{array}\right)}{\left(\begin{array}{c}
2 \\
i+j
\end{array}\right)} .
\end{aligned}
$$

Let $Q_{n}$ be the $(n+1) \times(n+1)$ matrix

$$
Q_{n}=\frac{1}{2 n+1}\left[\frac{\left(\begin{array}{c}
n \\
i
\end{array}\right)\left(\begin{array}{l}
n \\
j
\end{array}\right)}{\left(\begin{array}{c}
2 n \\
i+j
\end{array}\right)}\right]_{(n+1) \times(n+1)}
$$

Then, the $L_{2}$ norm of the Bézier curve $c^{n}$ is

$$
\left\|c^{n}\right\|_{2}^{2}=C^{t} Q_{n} C
$$


The matrix $Q_{n}$ is a real symmetric matrix. The following lemma gives equivalent conditions for the real symmetric matrix to be positive definite [16].

LEMMA 1. Each of the following tests is a necessary and sufficient condition for the real symmetric matrix $A$ to be positive definite:

1. $\mathrm{x}^{t} A \mathrm{x}>0$ for all nonzero vectors $\mathrm{x}$.

2. All the eigenvalues of $A$ are positive.

3. All the upper left submatrices have positive determinants.

From the definition of the matrix $Q_{n}$ and mathematical induction, all the upper left submatrices of the matrix $Q_{n}$ have positive determinants. Hence, the matrix $Q_{n}$ is real symmetric positive definite.

Thus, we obtain the following theorem for the $L_{2}$ distance between the Bézier curve $a^{n}$ of degree $n$ and the Bézier curve $b^{m}$ of degree $m$.

ThEOREM 2. The $L_{2}$ distance between the two Bézier curves $a^{n}$ and $b^{m}$ is

$$
d_{2}\left(a^{n}, b^{m}\right)=d_{2}\left(a^{n}, b^{(r)}\right)=\sqrt{D^{t} Q_{n} D}
$$

where $D=A-T_{m, r} B$ and $A=\left(a_{0}, \ldots, a_{n}\right)^{t}$ and $B=\left(b_{0}, \ldots, b_{m}\right)^{t}$.

The sum of any row and any column of the matrix $Q_{n}$ are both equal to $1 /(n+1)$, that is,

$$
\sum_{j=0}^{n} q_{i, j}=\sum_{i=0}^{n} q_{i, j}=\frac{1}{n+1} .
$$

\section{Degree Reduction}

By replacing the distance function $d$ in Problem 1 and writing the distance $d_{2}$ as in (4), Problem 1 can be rewritten as the following:

Problem 2. ( $L_{2}$ Degree Reduction.) Find another point set $\left\{c_{i}\right\}_{i=0}^{m}$ so that the least squares distance

$$
d_{2}\left(b^{n}, c^{m}\right)=d_{2}\left(b^{n}, c^{(r)}\right)=\sqrt{D^{t} Q_{n} D}
$$

between $\left\{b_{i}\right\}_{i=0}^{n}$ and $\left\{c_{i}^{(r)}\right\}_{i=0}^{n}$ is minimised.

Note that $T_{m, r} C=C^{(r)}$ and $B-C^{(r)}=D$ where $D=\left(d_{0}, d_{1}, \cdots, d_{n}\right)^{t}, d_{i}=b_{i}-c_{i}^{(r)}, i=$ $0,1, \cdots, n$.

For developing the method, rewrite $D^{t} Q_{n} D$,

$$
\begin{aligned}
D^{t} Q_{n} D & =\left[B-C^{(r)}\right]^{t} Q_{n}\left[B-C^{(r)}\right] \\
& =\left[B-T_{m, r} C\right]^{t} Q_{n}\left[B-T_{m, r} C\right] \\
& =B^{t} Q_{n} B-2 C^{t} T_{m, r}^{t} Q_{n} B+C^{t} T_{m, r}^{t} Q_{n} T_{m, r} C
\end{aligned}
$$


One method of obtaining the vector $C$ is the method of least squares. This method consists of minimising $D^{t} Q_{n} D$ with respect to $C$. Choosing the vector $\hat{C}$ as that value of $C$ which minimises $D^{t} Q_{n} D$ involves differentiating $D^{t} Q_{n} D$ with respect to the elements of $C$. On equating $\partial\left(D^{t} Q_{n} D\right) / \partial C$ to zero and writing the resulting equations in terms of $\hat{C}$, we find that these equations are

$$
T_{m, r}^{t} Q_{n} T_{m, r} \widehat{C}=T_{m, r}^{t} Q_{n} B
$$

They are known as the normal equations [12].

From the definition of the matrix $T_{m, r}$ and $Q_{n}$, we have that the matrix product $T_{m, r}^{t} Q_{n} T_{m, r}$ is $Q_{m}$. Hence, the real symmetric positive definite matrix $T_{m, \tau}^{t} Q_{n} T_{m, r}=Q_{m}$ is invertible. Provided $\left(T_{m, r}^{t} Q_{n} T_{m, r}\right)^{-1}$ exists, we have the unique solution for $\widehat{C}$,

$$
\widehat{C}=\left(T_{m, r}^{t} Q_{n} T_{m, r}\right)^{-1} T_{m, r}^{t} Q_{n} B .
$$

The approximate curve by using (6) is the best approximation with respect to the $L_{2}$ norm. The $L_{2}$ best approximation is the Legendre polynomials. See Eck [7] for detailed discussion.

For an error analysis, we need the definition of the Moore-Penrose inverse.

Definition 1: Let $A$ be the $n \times m$ matrix $(n>m)$. The $m \times n$ matrix $X$ is called the Moore-Penrose inverse of the $A$, if it satisfies the following conditions known as the Moore-Penrose conditions:

$$
\begin{array}{ll}
A X A=A, & (A X)^{t}=A X \\
X A X=X, & (X A)^{t}=X A .
\end{array}
$$

The Moore-Penrose inverse of $A$ is usually denoted by $A^{+}$. The Moore-Penrose inverse $A^{+}$of the matrix $A$ is uniquely determined [13]. If $\operatorname{rank}(A)=m$, then $A^{+}=\left(A^{t} A\right)^{-1} A^{t}$, while if $\operatorname{rank}(A)=m=n$, then $A^{+}=A^{-1}[\mathbf{1 3}]$.

The matrix $M=\left(T_{m, r}^{t} Q_{n} T_{m, r}\right)^{-1} T_{m, r}^{t} Q_{n}$ is the Moore-Penrose inverse of the matrix $T_{m, r}$.

To obtain the approximation error $\varepsilon_{L_{2}}$, put $\hat{C}$ into equation (5).

THEOREM 3. The error of the solution $\widehat{C}$ of the Problem 2 is

$$
\varepsilon_{L_{2}}^{2}=B^{t} Q_{n}\left[I-T_{m, r}\left(T_{m, r}^{t} T_{m, r}\right)^{-1} T_{m, r}^{t}\right] B
$$

For simple computation we may use the discrete $l_{2}$ distance function. Then the Problem 1 may be rewritten as the following:

Problem 3. ( $l_{2}$ Degree Reduction) Find control points $\left\{c_{i}\right\}_{i=0}^{m}$ so that the distance

$$
d_{D L S}\left(b^{n}, c^{m}\right)=d_{D L S}\left(b^{n}, c^{(r)}\right)=\sqrt{D^{t} D}
$$

between $\left\{b_{i}\right\}_{i=0}^{n}$ and $\left\{c_{i}^{(r)}\right\}_{i=0}^{n}$ is minimised. 
As in the case of $L_{2}$ degree reduction, we obtain the solution $\hat{C}_{D L S}$ as

$$
\widehat{C}_{D L S}=\left(T_{m, r}^{t} T_{m, r}\right)^{-1} T_{m, r}^{t} B
$$

The matrix $\left(T_{m, r}^{t} T_{m, r}\right)^{-1} T_{m, r}^{t}$ is also the Moore-Penrose inverse of $T_{m, r}$. By the uniqueness of the Moore-Penrose inverse, the $L_{2}$ solution $\widehat{C}$ and the discrete $l_{2}$ solution $\widehat{C}_{D L S}$ are equal, that is,

$$
\widehat{C}=\widehat{C}_{D L S}
$$

Thus the $L_{2}$ degree reduction curve and the discrete $l_{2}$ degree reduction curve are same.

To obtain the approximation error $\varepsilon_{l_{2}}$, put $\hat{C}_{D L S}$ into the equation in Problem 3.

THEOREM 4. The error of the solution $\widehat{C}_{D L S}$ of Problem 3 is

$$
\varepsilon_{l_{2}}^{2}=B^{t}\left[I-T_{m, r}\left(T_{m, r}^{t} T_{m, r}\right)^{-1} T_{m, r}^{t}\right] B
$$

The $(m+2) \times(m+2)$ matrix $P_{m}=I-T_{m}\left(T_{m}^{t} T_{m}\right)^{-1} T_{m}^{t}=\left\{p_{i, j}\right\}$ has elements

$$
p_{i, j}=(-1)^{i+j} \frac{\left(\begin{array}{c}
m+1 \\
i
\end{array}\right)\left(\begin{array}{c}
m+1 \\
j
\end{array}\right)}{\left(\begin{array}{c}
2 m+2 \\
m+1
\end{array}\right)}, i, j=0,1, \ldots, m+1 \text {. }
$$

\section{REFERENCES}

[1] P.Bézier, Numerical control, mathematics and applications (Wiley, New York, 1972).

[2] P. de Casteljau, Outillage méthodes calcul (André Citroën Automobiles SA, Paris, 1959).

[3] L. Dannenberg and H. Nowacki, 'Approximate conversion of surface representations with polynomial bases', Comput. Aided Geom. Design 2 (1985), 123-131.

[4] W.L.F. Degen, 'Best approximation of parametric curve by splines', in Mathematical methods in computer aided design II, (T. Lyche and L.L. Schumaker, Editors) (Academic Press, New York, 1992).

[5] J. Dugundji, Topology (Allyn and Bacon, Boston, 1966).

[6] M. Eck, 'Degree reduction of Bézier curves', Comput. Aided Geom. Design 10 (1993), 237-251.

[7] M. Eck, 'Least squares degree reduction of Bézier curves', Comput. Aided Geom. Design 27 (1995), 845-851.

[8] J.D. Emery, 'The definition and computation of a metric on plane curves', Comput. Aided Geom. Design 18 (1986), 25-28.

[9] G. Farin, 'Algorithms for rational Bézier curves', Comput. Aided Geom. Design 15 (1983), 73-77.

[10] G. Farin, Curves and surfaces for computer aided geometric design (Academic Press, New York, 1988).

[11] A.R. Forrest, 'Interactive interpolation and approximation by Bézier polynomials', Comput. Aided Geom. Design 22 (1990), 527-537.

[12] R. Gonin and A.H. Money, Nonlinear $L_{p}$-norm estimation (Marcel Dekker, Inc., New York, 1989). 
[13] G.H. Golub and C.F. Van Loan, Matrix computations (Johns Hopkins Univ. Press, Maryland, 1983).

[14] J. Hoscheck, 'Approximate conversion of spline curves', Comput. Aided Geom. Design 4 (1987), 59-66.

[15] M.A. Lachance, 'Chebyshev economization for parametric surfaces', Comput. Aided Geom. Design 5 (1988), 195-208.

[16] G. Strang, Linear algebra and its applications, (second edition) (Academic Press, New York, 1980).

[17] M.A. Watkins and J.A. Worsey, 'Degree reduction of Bézier curves', Comput. Aided Geom. Design 20 (1988), 398-405.

[18] Y. Park and U.J. Choi, 'The error analysis for degree reduction of Bézier curves', Comput. Math. Appl. 27 (1994).

[19] Y. Park, U.J. Choi and H.J. Kimn, 'Approximate conversion of Bézier curves', Bull. Austral. Math. Soc. 51 (1995), 153-162.

Department of Applied Mathematics

Dongseo University

Pusan, 617-716

Korea
Department of Mathematics Education

Seowon University

Chongju, 361-742

Korea 